\title{
REFINED CALCULATION OF BEAM DYNAMICS DURING UMER INJECTION *
}

\author{
G. Bai", R.A. Kishek, S. Bernal, T. Godlove, I. Haber, B. Quinn, M. Reiser, C. Tobin, M. Walter, \\ and P.G. O'Shea
}

\author{
Institute for Research in Electronics and Applied Physics \\ University of Maryland, College Park, MD 20742
}

\begin{abstract}
The University of Maryland Electron Ring (UMER) is built as a low-cost testbed for intense beam physics for benefit of larger ion accelerators [1]. The beam intensity is designed to be variable, spanning the entire range from low current operation to highly space-charge-dominated transport. The ring has recently been closed and multiturn commissioning has begun. Although we have conducted many experiments at high space charge during UMER construction, lower-current beams have become quite useful in this commissioning stage for assisting us with beam steering, measurement of phase advance, etc. One of the biggest challenges of multi-turn operation of UMER is correctly operating the Y-shaped injection section, hence called the Y-section, which is specially designed for UMER multi-turn operation. It is a challenge because the system requires several quadrupoles and dipoles in a very stringent space, resulting in mechanical, electrical, and beam control complexities. This paper presents a simulation study of the beam centroid motion in the injection region.
\end{abstract}

\section{INTRODUCTION}

UMER is a low-cost, scaled, electron storage ring designed for research in beam physics in order to benefit larger ion machines [1]. At a rep. rate of $60 \mathrm{~Hz}$, a single long $100 \mathrm{~ns}$ pulse is injected into UMER from a thermionic gridded gun at an energy of $10 \mathrm{keV}$ and currents ranging from $0.6-100 \mathrm{~mA}$. Since this pulse is long enough to occupy half the ring, we have a window of less than $100 \mathrm{~ns}$ after injection to flip the polarity of the single pulsed dipole, $\mathrm{PD}$, in times for recirculating the injected pulse. Furthermore, due to the paucity of space and the short lattice period, a single quadrupole, YQ, is shared between the injection line and the returning part of the ring. Figure 1 is a Pro-E drawing of injector section (Y-section), used for injection. It is composed of an injection leg (flange upstream Q5 to PD), joining a returning leg (flange upstream Q70 to PD), each making a $10^{\circ}$ angle with the ring part (PD to the flange downstream QR1). The shared quadrupole, YQ, is off-centered from the reference trajectories of the beam, hence plays a part in bending the beam towards the injector. Additional steering dipoles, $\mathrm{SD}$, are added upstream to help steer the beam.

*Work supported by US Department of Energy grant numbers: DEFG02 -94ER40855 and DE-FG02-92ER54178.

"gbai@umd.edu
The primary difficulty in this design is being able to correctly set the steering dipoles and the pulsed dipole for optimal steering. In the experiment the entire Y-section is blind (i.e. has no diagnostics), making beam-based steering difficult. In addition the UMER beam energy is so low making the beam susceptible to the Earth magnetic field, which is unshielded in the region of the Y. In Maryland, the Earth magnetic field is of the order of 0.5 Gauss mostly pointing vertically down, and can thus bend the beam considerably over the 64-cm length of the Ysection, in addition to breaking the symmetry between injection and return current since the Earth field favors only one direction. Our existing models of the Y-section, both the one based on transfer matrices, as well as the WARP models with detailed magnet representations [2], ignored the earth field. In addition, the transfer matrix calculation considered the pulsed dipole as a thin dipole.

In this paper, we construct a more realistic model that includes the effects of the earth field as well as represents the pulsed dipole as thick dipole with a nonzero effective length. These complications, however, make the transfer matrix of the Y-section much harder to derive analytically. We therefore rely more on this code to generate the theoretical dipole settings, and are currently working on integrating it with UMER control software.

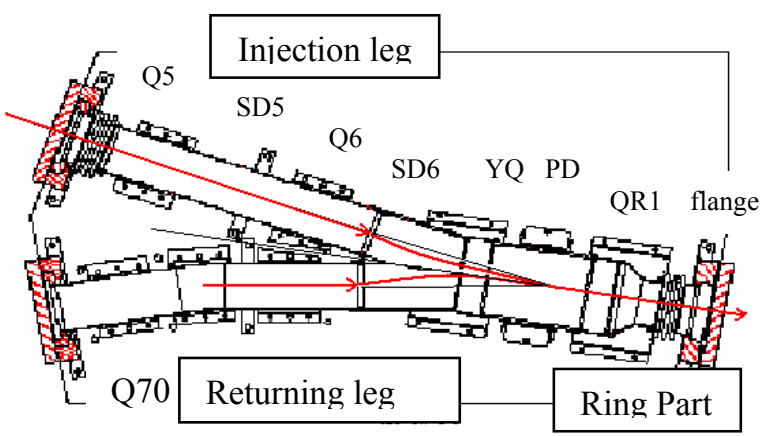

Figure 1: UMER injection section

\section{SIMPLE MODEL CALCULATION}

The "simple model" used earlier here consists only of the central part of the Y-section, and is composed of one big DC Quadrupole (we call it YQ), the Pulsed Dipole (PD) and one steering dipole in the injection line (SD6). It improves over the simpler model presented in Ref. [3] in which it tracks the beam trajectory under the effects of both the earth field and the PD, in addition to the YQ in order to obtain the bend angles that need to be applied to 
SD6 and the pulsed dipole respectively for centering the beam. A code written in MATLAB tracks the beam trajectory in a two-dimension plane $x-z$, where $z$ is beam propagation direction after the pulsed dipole, and $x$ is perpendicular to that in the horizontal plane (the bend plane). Note that motion in the $x-z$ plane is affected by the vertical component of the Earth field. This new model is shown in Figure 2.

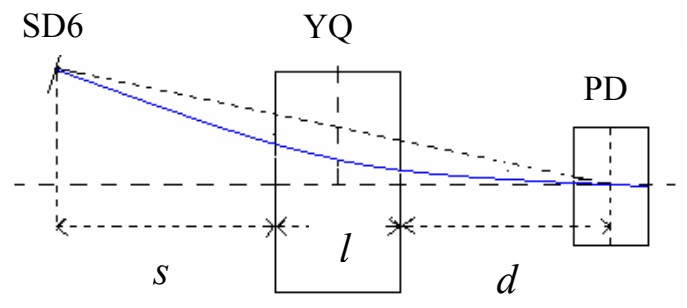

Figure 2: Simple model

Here, the YQ effective length $l=5.38 \mathrm{~cm}$, the Pulsed Dipole effective length is $5.18 \mathrm{~cm}, s=9.0 \mathrm{~cm}, d=5.31$ $\mathrm{cm}$, and the gradient of YQ is fixed as $5.33 \mathrm{G} / \mathrm{cm}$. We make the center of big quadrupole (YQ) the origin ( $x=0$, $z=0$ ) of our simulation model.

The main idea of our MATLAB algorithm is to successively iterate the bend angle of SD6 and the magnetic field strength of the Pulsed Dipole to make both the x-offset of the centroid and its exit angle from the pulsed dipole zero at the right edge of Pulsed Dipole. We assume beam enters SD6 perfectly aligned, meaning oncenter with a zero incidence angle.

To check the effect of the finite effective length of PD, we run this new model without the earth field, positioning the thin dipole in the center of our wide PD; run previous calculation [3] with the same parameters as the new model. The results of this comparison are shown in Table 1 , demonstrating fairly good predictions using the thindipole model used earlier.

Table 1: Comparison with previous work

\begin{tabular}{|c|c|c|}
\hline & SD6 angle & PD angle \\
\hline Previous Model & $2.08^{\circ}$ & $7.13^{\circ}$ \\
\hline Current Model & $2.156^{\circ}$ & $7.066^{\circ}$ \\
\hline
\end{tabular}

For finding the angles of SD6 and the PD, we develop two iterative procedures (A and $\mathrm{B}$ ). In procedure $\mathrm{A}$, we scan the bending angle of SD6 until the beam is centered in the PD. We get an SD6 angle of $2.136^{\circ}$, and a PD field of 7.8 Gauss, corresponding to an angle of $6.985^{\circ}$. In procedure $\mathrm{B}$, the magnetic field strength of Pulsed Dipole is scanned to get the same goal. The results obtained from procedure $\mathrm{B}$ agree with procedure $\mathrm{A}$ well.

To assess the effect of earth field, we add a uniform field over the entire area equal to 0.5 Gauss. This is in addition to the wide PD model. Similarly, we run procedure A and B to get converging results, with an SD6 bending angle of $1.435^{\circ}$ and a PD magnetic field strength 8.9 Gauss, which is a significant difference that can result in serious mis-steering if not properly accounted for. The effects of earth field on beam trajectory are displayed in Figure 3.

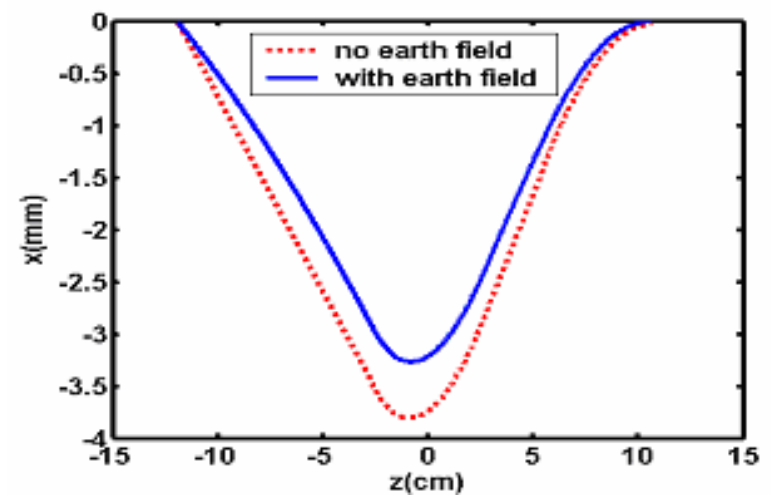

Figure 3: Beam trajectory: wide PD, with/without Earth field

Table 2 compares the results of different models and indicates the effects of considering earth field on designing the optics in injection section.

Table 2: Comparison of key lenses values in three models

\begin{tabular}{|c||c|c|c|c||c|c|c|}
\hline \multicolumn{1}{|c||}{} & \multicolumn{3}{|c|}{ SD6 } & \multicolumn{3}{c|}{ Pulsed Dipole (PD) } \\
\cline { 2 - 8 } & $\begin{array}{c}\theta \\
(\mathrm{deg})\end{array}$ & $\begin{array}{c}\text { Current } \\
(\mathrm{A})\end{array}$ & $\begin{array}{c}\mathrm{S} \\
(\mathrm{G})\end{array}$ & $\begin{array}{c}\beta \\
(\mathrm{deg})\end{array}$ & $\begin{array}{c}\text { Current } \\
(\mathrm{A})\end{array}$ & $\begin{array}{c}\mathrm{S} \\
(\mathrm{G})\end{array}$ & $\begin{array}{c}\mathrm{S} \\
(\mathrm{G})\end{array}$ \\
\hline Thin PD & 2.156 & 3.499 & 4.1 & 7.066 & 20.797 & - & 8.0 \\
\hline W-PD & 2.136 & 3.537 & 4.2 & 6.985 & 20.973 & 7.8 & 8.1 \\
\hline $\begin{array}{c}\text { W-PD + } \\
\text { Be }\end{array}$ & 1.435 & 2.376 & 2.8 & 7.267 & 23.119 & 8.9 & 8.9 \\
\hline
\end{tabular}

$\theta$ is bending angle by SD6 while $\beta$ is the angle of PD. SD6 int. gradient is $3.627 \mathrm{G} \cdot \mathrm{cm} / A$, effective length is $3.063 \mathrm{~cm}$; PD int. gradient is $2 \mathrm{G} \cdot \mathrm{cm} / \mathrm{A}$ and effective length is $5.18 \mathrm{~cm}$. W-PD is Wide $\mathrm{PD}$; $\mathrm{S}$ is magnet strength; $S^{\prime}$ is theoretical calculations, which agree with the results obtained from simulation $\mathrm{S}$.

\section{INJECTION SECTION SIMULATION}

In the former simulation, we track the beam so that the beam centroid would have zero x-offset and zero angle at the right edge of Pulsed Dipole. In experiments, we find it is difficult to realize it. To see what happens, we run WARP simulation of beam centroid trajectory in the whole ring. The entire ring is composed of 36 FODO (a ring dipole, a focusing quadrupole and a defocusing quadrupole separated by the ring dipole). The simulation idea is: running beam from beginning point just before YQ with initial position and angle $\left(x_{0}, x_{0}^{\prime}\right)$; after one turn, we make beam hit the beginning point at same position and angle, $\left(x, x^{\prime}\right)=\left(x_{0}, x_{0}^{\prime}\right)$. The WARP simulation results are shown in Figure 4. [4] 


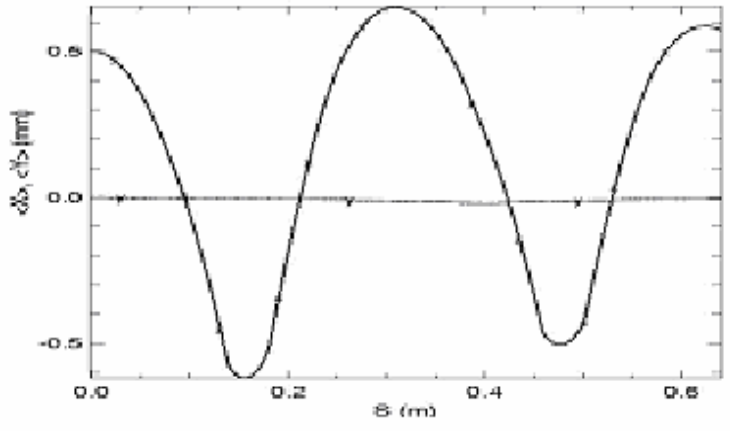

Figure 4: Beam centroid trajectory in UMER

Beam centroid transports with a periodic fluctuation along the ring. $x$ offsets at ring dipoles fluctuate at dipoles around $0.5 \mathrm{~mm}$. This beam trajectory gives us estimated beam centroid positions under the effects of periodically set dipoles and Earth field, on which specific simulation of beam trajectory in UMER injection is done.

The injection section model is displayed in Figure 5, composed by injection leg part and central Y section (SD4 to $\mathrm{PD}$ ), the ring part (PD to the flange downstream QR1).

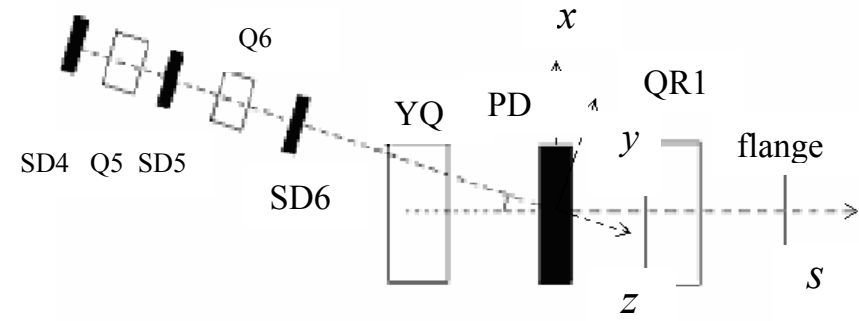

Figure 5: Injection section model

We use a new idea to develop the simulation algorithm of beam trajectory. The idea is based on beam transportation equations. If beam transports in a 2-D system $s-x$, shown in Figure 5, $s$ is beam transportation direction, we have the following equations:

$$
\begin{aligned}
& x^{\prime \prime}=\ddot{x} / v^{2} \\
& x^{\prime \prime}=\frac{-q B_{e}}{m v} \quad \text { (1) } \quad v \text { is beam velocity; } B_{e} \text { is earth field } \\
& x^{\prime \prime}=\frac{d x^{\prime}}{d s}=\frac{x_{i}^{\prime}-x_{i-1}^{\prime}}{d s} \text { (4) } x_{i}^{\prime}=x_{i-1}^{\prime}+\left(\frac{-q B_{e}}{m v}\right) \cdot d s \text { (5) } \\
& x_{i}^{\prime}=\frac{d x}{d s} \quad x_{i}=x_{i-1}+x_{i}^{\prime} \cdot d s \\
& \operatorname{tg} \theta_{i}=\frac{v_{x}}{v_{s}}=\frac{d x / d t}{d s / d t}=\frac{d x}{d s}=x_{i}^{\prime}(8) \quad s_{i}=s_{i-1}+d s
\end{aligned}
$$

(1)
considerations of key lens and earth field, we have compared the simulation results and see the effects of earth field, which should not be ignored. The effect of earth field is accounted in this model, making it difficult to calculate beam position and angle with traditional transformation matrix. We have tried to track beam trajectory in the interested region to obtain currents or deflection angles of several important lenses involved, which guide future experiments.

\section{REFERENCES}

[1] S. Bernal, et al, Commissioning of the University of Maryland Electron Ring (UMER), these proceedings, PAC'2005, Knoxville, TN

[2] D.P. Grote, et al, Fus. Eng. Des. 32-33,193-200, 1996

[3] H. Li, et al, Beam Optics Design on a New Injection Scheme for UMER, PAC'2003, Portland, OR

[4] R.A. Kishek, et al, Significance of the Earth Magnetic Field on Dispersive Characteristics of a Low Energy Electron Beam, PAC'2005, Knoxville, TN 